\title{
A BIORTHOGONAL SYSTEM WHICH IS NOT A TOEPLITZ BASIS
}

\author{
BY ALBERT WILANSKY AND KARL ZELLER
}

Communicated by Edwin Hewitt, June 7, 1963

We present a Banach space $X$ with a biorthogonal system $\left\{b_{n}, f_{n}\right\}$, $b_{n} \in X, f_{n} \in X^{*}, f_{i}\left(b_{j}\right)=\delta_{i j}$, in which $\left\{b_{n}\right\}$ is fundamental, i.e., $X$ is its linear closure, but $\left\{b_{n}\right\}$ is not a Toeplitz basis. We call $\left\{b_{n}\right\}$ a Toeplitz basis if there exists a regular matrix $A$ such that for each $x \in X, \sum f_{n}(x) b_{n}$ is $A$-summable to $x$. It will be clear that $\left\{b_{n}\right\}$ is not a Toeplitz basis even if the method of summability is considerably more general than a matrix.

An example in which $X$ is a non-Banach $F$ space is given in [4, Theorem 13]. Toeplitz bases were introduced in [1], [2].

A series $\sum u_{n}$ is said to be weakly Cauchy if for every continuous linear functional $f, \sum f\left(u_{n}\right)$ is convergent.

Lemma 1. Let $X$ be a linear topological space with a biorthogonal system $\left\{b_{n}, f_{n}\right\}$. Suppose that there exists $x_{0} \in X$ such that $\sum f_{n}\left(x_{0}\right) b_{n}$ is weakly Cauchy. Then if $\left\{b_{n}\right\}$ is a Toeplitz basis, $\sum f_{n}\left(x_{0}\right) b_{n}$ must converge weakly to $x_{0}$.

For any $f \in X^{*}, \sum f_{n}\left(x_{0}\right) f\left(b_{n}\right)$ is convergent. It must converge to $f\left(x_{0}\right)$ since, for some regular matrix $A$, it is $A$-summable to $f\left(x_{0}\right)$.

The full force of the hypothesis is not used. It can be considerably weakened.

From Lemma 1 it follows that the promised example will be fulfilled by an example of a Banach space $X$ of sequences $x=\left\{x_{n}\right\}$ in which, for each $n, f_{n}(x)=x_{n}$ defines $f_{n} \in X^{*}$; in which $\left\{\delta^{n}\right\}$ is fundamental, where $\delta^{1}=(1,0,0, \cdots), \delta^{2}=(0,1,0, \cdots), \cdots$; in which $\sum \delta^{n}$ is weakly Cauchy; but in which $\sum \delta^{n}$ does not converge weakly to 1 .

Let $B=\left(b_{n k}\right)$ be a matrix which is a triangle, i.e., $b_{n n} \neq 0, b_{n k}=0$ for $k>n$; which is coregular, i.e., $\chi(B) \equiv \lim _{B} 1-\sum \lim _{B} \delta^{k} \neq 0$ where $\lim _{B} x=\lim _{n} \sum_{k} b_{n k} x_{k}$; for which $\left\{\delta^{n}\right\}$ is fundamental in $c_{B}$ $=\{x: x$ is $B$ summable $\},\|x\|=\sup _{n}\left|\sum_{k} b_{n k} x_{k}\right| ;$ but which has the property that no regular matrix $D$ has $c_{D}=c_{B}$. (See $[3$, p. 657] for an example.)

Then $\sum \delta^{n}$ is weakly Cauchy since $c_{B}$ has a weaker topology than that of $c_{0}$, the space of null sequences with $\|x\|=\sup \left|x_{n}\right|$, and, in the latter space $\sum \delta^{n}$ is weakly Cauchy.

If $\left\{\delta^{n}\right\}$ were a Toeplitz basis, by Lemma 1 , we should have $\sum \delta^{n}$ 
converging weakly to 1 . Since $\lim _{B} \in c_{B}^{*}$ this would yield $\chi(B)=0$, contradicting the fact that $B$ is coregular.

Since $c_{B}$ is congruent with $c$, the space of convergent sequences with $\|x\|=\sup \left|x_{n}\right|$ we have proved the following result.

THEOREM 1. The space $c$ has a biorthogonal system $\left\{b_{n}, f_{n}\right\}$ in which $\left\{b_{n}\right\}$ is fundamental but is not a Toeplitz basis.

\section{REFERENCES}

1. B. R. Gelbaum, Expansions in Banach spaces, Duke Math. J. 17 (1950), 187196.

2. V. Y. Kozlov, On a generalization of the concept of basis, Dokl. Akad. Nauk SSSR 73 (1950), 643-646.

3. A. Wilansky, Summability: the inset, replaceable matrices, the basis in summability space, Duke Math. J. 19 (1952), 647-660.

4. A. Wilansky and $\mathrm{K}$. Zeller, The inverse matrix in summability: reversible matrices, J. London Math. Soc. 32 (1957), 397-408.

\section{LEHIGH UNIVERSITY AND}

TÜBINGEN UNIVERSITY 